\title{
Salvage Photodynamic Therapy Is an Effective and Safe Treatment for Patients with Local Failure after Definitive Chemoradiotherapy for Esophageal Squamous Cell Carcinoma
}

\author{
Yoko Mashimo1, Yasumasa Ezoe' ${ }^{2}$, Kosuke Ueda', Yoshinao Ozaki', Yusuke Amanuma', \\ Ikuo Aoyama', Takahiro Horimatsu3 ${ }^{3}$, Shuko Morita'2, Shin'ichi Miyamoto1, \\ Tomonori Yano4, Koji Higashino5, Tsutomu Chiba1, Manabu Muto² \\ ${ }^{1}$ Department of Gastroenterology and Hepatology, Graduate School of Medicine, Kyoto University, Kyoto, Japan \\ ${ }^{2}$ Department of Therapeutic Oncology, Graduate School of Medicine, Kyoto University, Kyoto, Japan \\ ${ }^{3}$ Department of Experimental Therapeutics, Institute for Advancement of Clinical and Translational Science \\ (iACT), Kyoto University Hospital, Kyoto, Japan \\ ${ }^{4}$ Division of Digestive Endoscopy and Gastrointestinal Oncology, National Cancer Center Hospital East, Kashiwa, \\ Japan \\ ${ }^{5}$ Department of Gastrointestinal Oncology, Osaka Medical Centre for Cancer and Cardiovascular Diseases, \\ Osaka, Japan \\ Email: mashimo@kuhp.kyoto-u.ac.jp
}

Received 11 February 2014; revised 10 March 2014; accepted 18 March 2014

Copyright (C) 2014 by authors and Scientific Research Publishing Inc.

This work is licensed under the Creative Commons Attribution International License (CC BY).

http://creativecommons.org/licenses/by/4.0/

(c) (i) Open Access

\section{Abstract}

Although chemoradiotherapy (CRT) is one curative treatment option for patients with esophageal squamous cell carcinoma (ESCC), local failure after CRT remains a major problem for patients' curability. The aim of this study was to evaluate the efficacy and safety of photodynamic therapy (PDT) as a salvage treatment for local failure. From August 2007 to March 2012, 193 consecutive ESCC patients were treated with definitive CRT in Kyoto University Hospital. Eighteen of the patients with T2 or earlier T-stage local failure after CRT underwent salvage PDT. After the salvage PDT, 11 patients $(61.1 \%)$ achieved a complete response at the primary site. Over a median followup period of 28.3 months, the 1-year survival and progression-free survival rates were $77.8 \%$ and $38.9 \%$, respectively; and the 2-year survival and progression-free survival rates were $60.6 \%$ and $33.3 \%$, respectively. Adverse events were mild esophageal stricture in seven (38.9\%), esophageal obstruction because of necrotic tissue in nine $(50.0 \%)$, and photosensitivity in three $(16.7 \%)$ patients. There were no severe complications or treatment-related deaths. Salvage PDT may be a 
promising treatment option for the patients with a local residual or recurrent tumor after CRT for ESCC when the lesion is suspected to be in T2 or earlier.

\title{
Keywords
}

\author{
Esophageal Cancer, Salvage Treatment, Photodynamic Therapy, Chemoradiotherapy, Local Failure
}

\section{Introduction}

Definitive chemoradiotherapy (CRT) is one curative treatment for esophageal squamous cell carcinoma (ESCC). However, the high local recurrence rate (40\%) [1] is one of the major problems, because a recurrent or residual local tumor in the primary site after CRT tends to grow rapidly and develop esophageal stricture resulting dysphagia in a short time. Appropriate salvage treatment for local failure after CRT is now required to improve both the patient's prognosis and quality of life. At present, salvage surgery or second-line chemotherapy is generally indicated for local failure after CRT. The treatment outcome of second-line chemotherapy for patients with treatment failure after definitive CRT is poor and a cure could not be expected (i.e., median survival time; 8.1 months and 1-year survival rate; 34\%) [2]. Thus, only a salvage esophagectomy has a high probability of achieving a long-term survival (5-year survival rate; 25\%) and providing a cure for patients with a T2 or earlier T-stage tumor or for patients without lymph node metastasis [3]. However, the morbidity and mortality rates of salvage esophagectomy are reported to be high [3]. Therefore, the establishment of minimally invasive and curative salvage treatment options for local failure is essential for improving the survival and quality of life of the patients treated with CRT. In addition, patients who achieve a complete response (CR) with CRT are very unlikely to experience a recurrence in locoregional lymph nodes (1\%) and distant metastasis (9\%) [4]. Therefore, a local treatment might have a curative potential for the patients with local failure after CRT. Endoscopic mucosal resection (EMR) or endoscopic submucosal dissection (ESD) as the salvage treatment for local failure after CRT has acceptable outcomes only for superficial mucosal lesions in the esophagus [5]; however, these procedures are not indicated for deeper lesions because of technical limitations. By contrast, photodynamic therapy (PDT) could be theoretically indicated for the esophageal cancer invading to the shallow layer of the muscularis propria in the esophagus [6]. Yano et al. applied PDT as a salvage local treatment (salvage PDT) and reported promising results [7] [8]. Nevertheless, at present, salvage PDT is not recognized widely as a salvage treatment for local failure after CRT. In this study, we retrospectively investigated the efficacy and the safety of PDT as a local salvage treatment for the patients with local failure after CRT for ESCC.

\section{Patients and Methods}

\subsection{Patients}

From August 2007 to March 2012, 193 consecutive patients with ESCC received CRT or RT as a first-line treatment with $>50$ Gy external beam irradiation at the Kyoto University Hospital, and their clinical records were reviewed retrospectively. Nineteen patients were excluded because of the lack of clinical information or discontinuation of follow-up. Remaining 174 patients were included in this study. The clinical course of the patients is shown in Figure 1. After completion of the first-line CRT or RT, 128 patients achieved a CR and 46 did not achieve a CR (non-CR). Forty-nine of the 128 patients who achieved a CR developed recurrence subsequently. Among the 95 patients who experienced first-line treatment failure (49 patients with recurrent disease and 46 patients with residual disease), 47 patients developed local failure at the primary site without distant failure such as metastasis to the lymph nodes or other organs. Four of these patients received a salvage esophagectomy, 13 received second-line chemotherapy, four received salvage endoscopic treatment (EMR/ESD), two received best supportive care, and the other 24 received salvage PDT. We performed salvage PDT using porfimer sodium as a photosensitizer and the excimer dye laser as the laser light for 18 patients in the clinical practice and for six patients with Laserphyrin and diode laser in a clinical trial. Finally, 18 patients who were treated with salvage PDT in the clinical practice were included in this study. All information was collected from medical records. Written informed consent was obtained from all patients before performing salvage PDT. 


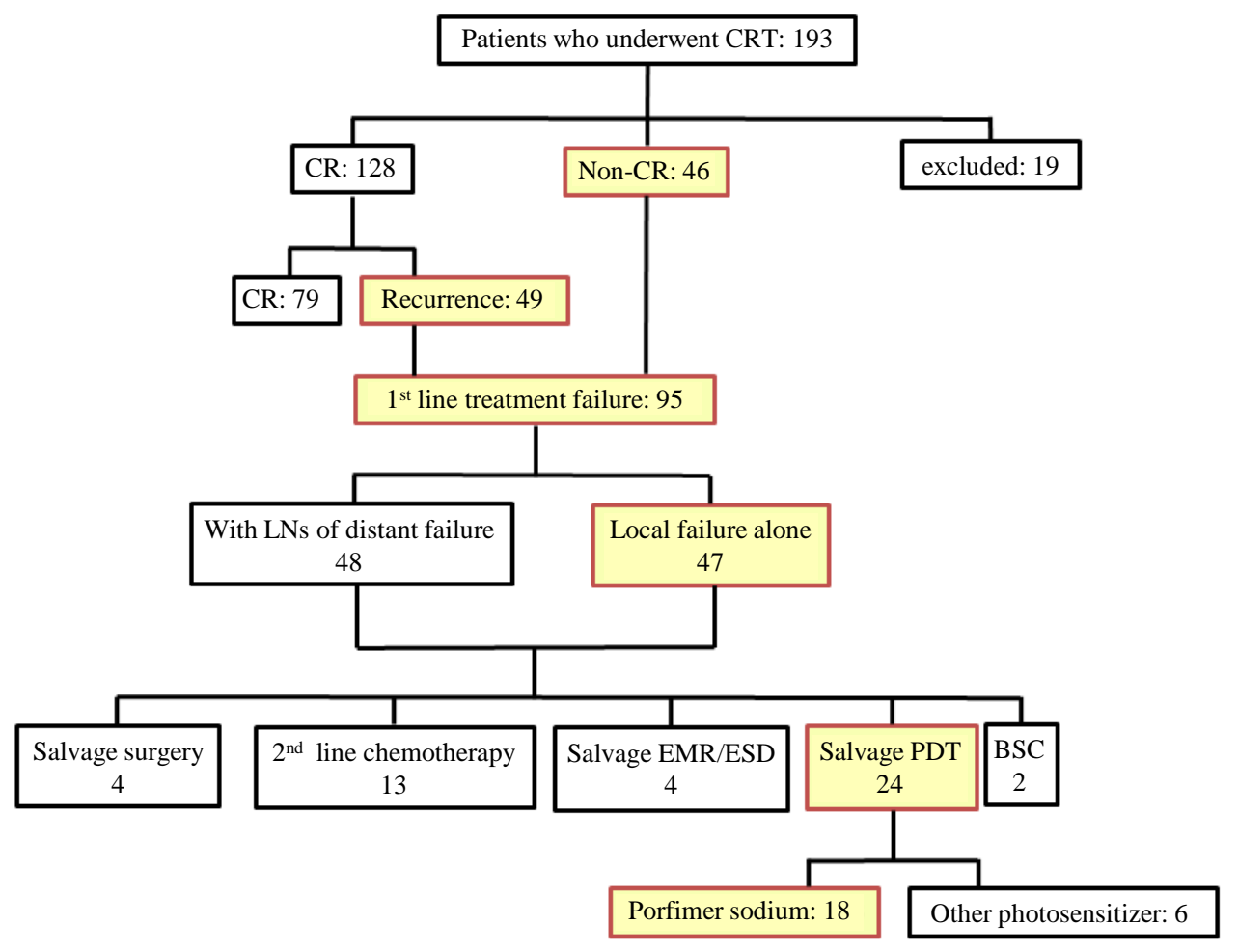

Figure 1. Clinical course of all patients who underwent chemoradiotherapy for esophageal squamous cell carcinoma (ESCC). CRT, chemoradiotherapy; CR, complete response; LN, lymph node; EMR, endoscopic mucosal resection; ESD, endoscopic submucosal dissection; PDT, photodynamic therapy; BSC, best supportive care.

\subsection{Indication of Salvage PDT}

Salvage PDT was indicated for patients who fulfilled the following criteria: 1) local failure at the primary site in the esophagus after definitive CRT; 2) clinical N0 M0 stage defined by computed tomography (CT) at the time of salvage, 3 ) if the lymph node or distant metastases were expected to be stable with other therapies, these patients were also regarded as eligible; 4) the depth of the local failure at the primary site was estimated to be within the submucosa (uT1b) or the muscularis propria (uT2) by endoscopic ultrasound (EUS); 5) the patient refused a salvage esophagectomy or the physical condition was poor and surgery was contraindicated; and 6) written informed consent had been provided.

\subsection{Identification and Evaluation of Locoregional Failure after Definitive CRT or Radiotherapy}

To detect local failures at an early stage, careful and close endoscopic surveillance were performed. Until the confirmation of a CR, the follow-up examinations using esophagogastroduodenoscopy (EGD) and CT were performed every month after the completion of CRT or RT. After confirmation of a CR, follow-up examinations were performed every 2 or 3 months for 2 years, and every 6 months thereafter. Pathological confirmation using biopsy specimens obtained from the primary site was performed for every follow-up endoscopic examination. If a suspicious lesion was detected, a detailed evaluation to confirm whether the tumor like lesion was detected and grade of the invasion by EUS (ultrasound system, EU-ME1; miniature probe, UM-S20-20R: Olympus Medical Systems Co. Ltd, Tokyo, Japan). Clinical staging was determined by the $7^{\text {th }}$ TNM classification of the International Union Against Cancer [9]. Clinical T stage was determined by considering all findings obtained by EGD, EUS, and CT. Clinical N and M stages were evaluated by CT of the neck, chest and abdomen. Lymph node metastases were diagnosed when the size of the lymph node was $>10 \mathrm{~mm}$ in diameter on CT. A CR at the primary site was defined as follows: 1) disappearance of the tumor lesion and ulceration as shown by EGD, and 2) histological absence of cancer cells in endoscopic biopsy specimens obtained from the primary site [10]. Local fail- 
ure was diagnosed when the primary site showed macroscopic progression with histological confirmation of cancer cell. In the case with macroscopic progression but without histological confirmation, the existence of the marginated heteroechoic solid component in the esophageal layer by EUS was considered to indicate local failure. Local failure was classified into a residual or recurrent lesion. A residual lesion was defined as a lesion that developed but never achieved CR after completion of CRT or RT. A recurrent lesion was defined as a lesion that had regrown after it had achieved CR.

\subsection{Salvage PDT}

We use a wavelength of $630 \mathrm{~nm}$ for the excimer dye laser (EDL-1, Hamamatsu Photonics, Hamamatsu, Japan) as the laser beam and $2 \mathrm{mg} / \mathrm{kg}$ of porfimer sodium as the photosensitizer. We irradiate the laser beam through the endoscope 48 and $72 \mathrm{~h}$ after the injection of the photosensitizer. Patients need to be shielded from bright light, especially sunlight to prevent photosensitivity for about 1 month after the injection of photosensitizer. We set up the laser beam at $75 \mathrm{~J} / \mathrm{cm}^{2}$ and irradiate the target lesion. We perform EGD once a week until the irradiated site becomes completely scarred to remove the necrotic tissue and dilate the irradiated site with the endoscopic balloon to prevent the formation of an esophageal stricture. The treatment schedule after the salvage PDT is shown in Figure 2.

\subsection{Statistics}

Statistical analysis was performed using SPSS software, version 20 (IBM Corp., Armonk, NY). Progression-free survival (PFS) was measured from the date of initial PDT to the first date of detection of a histologically proven residual or recurrent lesion at the primary site, disease progression at any site, or the patient's death. Overall survival (OS) was measured from the date of initial PDT to the date of the patient's death for any reason or the date of the patient's last follow-up visit. Survival time was calculated by the Kaplan-Meier method.

\section{Results}

\subsection{Patients' Characteristics}

Patients' baseline characteristics before first-line CRT and before salvage PDT are summarized in Table 1. The patients included 14 men and four women with a median age of 70.8 years (range 54 - 83 years). All 18 patients had histologically proven ESCC before CRT. Thirteen patients were confirmed histologically to have recurrent or residual ESCC before PDT and five patients were assessed as having local failure clinically by EGD and EUS. In all patients, the depth of the recurrent or residual lesion was estimated by EUS to be within the submucosa (uT1b) or the muscularis propria (uT2). The clinical TNM stages [9] before salvage PDT were N0 M0 in 16 pa-

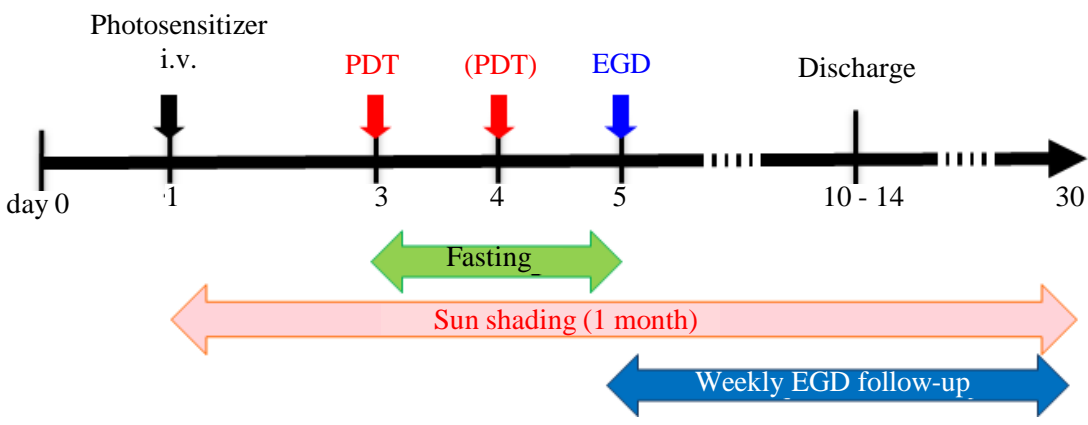

Figure 2. Treatment schedule of salvage photodynamic therapy (PDT). The excimer dye laser is irradiated at $48 \mathrm{~h}$ (and at $72 \mathrm{~h}$ if the additional irradiation is needed) after the intravenous administration of $2 \mathrm{mg} / \mathrm{kg}$ of photosensitizer (Porfimer sodium). Patients need to fast for 1 day (or 2 days if the additional irradiation is needed) and to avoid direct exposure to sunlight to prevent photosensitivity for 1 month after the injection of photosensitizer. We perform esophagogastroduodenoscopy (EGD) once a week until the irradiated site becomes completely scarred to remove the necrotic tissue, and we use on-demand endoscopic balloon dilatation to prevent the development of stricture. 
Table 1. Patients’ characteristics and outcomes of salvage PDT.

\begin{tabular}{|c|c|c|c|c|c|c|c|c|c|c|c|}
\hline \multirow[b]{2}{*}{ Patient } & \multirow[b]{2}{*}{ Age } & \multirow[b]{2}{*}{$\begin{array}{l}\text { Sex } \\
(\mathrm{M} / \mathrm{F})\end{array}$} & \multirow[b]{2}{*}{$\begin{array}{c}\text { Baseline } \\
\text { TNM } \\
\text { stage }\end{array}$} & \multirow[b]{2}{*}{$\begin{array}{l}\text { Best } \\
\text { response } \\
\text { for CRT }\end{array}$} & \multirow[b]{2}{*}{$\begin{array}{c}\text { TNM } \\
\text { stage } \\
\text { before } \\
\text { PDT }\end{array}$} & \multirow[b]{2}{*}{$\begin{array}{l}\text { Best } \\
\text { response } \\
\text { for PDT }\end{array}$} & \multicolumn{3}{|c|}{ Adverse event (CTCAE) ${ }^{*}$} & \multirow[b]{2}{*}{ Recurrence } & \multirow[b]{2}{*}{ Outcome } \\
\hline & & & & & & & $\begin{array}{l}\text { Esophageal } \\
\text { stricture }\end{array}$ & $\begin{array}{c}\text { Esophageal } \\
\text { obstruction } \\
\text { by necrotic } \\
\text { tissue }\end{array}$ & Photosensitivity & & \\
\hline 1 & 79 & M & T4 N0 M0 & Non-CR & T2 N0 M0 & Non-CR & 1 & 2 & 0 & Primary site & $\begin{array}{l}\text { Death from } \\
\text { ESCC }\end{array}$ \\
\hline 2 & 71 & $\mathrm{~F}$ & T4 N1 M0 & CR & T2 N1 M0 & Non-CR & 0 & 1 & 0 & Primary site & $\begin{array}{l}\text { Death from } \\
\text { ESCC }\end{array}$ \\
\hline 3 & 74 & M & T3 N1 M1b & CR & $\begin{array}{l}\text { T1b N0 } \\
\text { M0 }\end{array}$ & Non-CR & 1 & 2 & 0 & Primary site & $\begin{array}{l}\text { Death from } \\
\text { ESCC }\end{array}$ \\
\hline 4 & 54 & M & T4 N1 M0 & CR & T2 N0 M0 & Non-CR & 0 & 0 & 0 & $\begin{array}{c}\text { Primary site, } \\
\text { LNs }\end{array}$ & $\begin{array}{l}\text { Death from } \\
\text { ESCC }\end{array}$ \\
\hline 5 & 73 & $\mathrm{~F}$ & T4 N1 M0 & CR & $\begin{array}{l}\text { T1b N0 } \\
\text { M0 }\end{array}$ & Non-CR & 0 & 1 & 0 & Primary site & $\begin{array}{l}\text { Death from } \\
\text { ESCC }\end{array}$ \\
\hline 6 & 61 & M & T4N1 M1b & Non-CR & $\begin{array}{l}\text { T1b N1 } \\
\text { M1b }\end{array}$ & CR & 0 & 0 & 0 & LNs & $\begin{array}{l}\text { Death from } \\
\text { ESCC }\end{array}$ \\
\hline 7 & 67 & M & T4 N1 M0 & Non-CR & T1 N0 M0 & CR & 1 & 2 & 2 & None & Alive \\
\hline 8 & 80 & M & T2 N1 M1b & CR & T2 N0 M0 & CR & 1 & 0 & 0 & Primary site & $\begin{array}{l}\text { Death from } \\
\text { ESCC }\end{array}$ \\
\hline 9 & 69 & M & T2 N1 M0 & CR & $\begin{array}{l}\text { T1b N0 } \\
\text { M0 }\end{array}$ & CR & 0 & 1 & 0 & None & $\begin{array}{l}\text { Death from } \\
\text { other cause }\end{array}$ \\
\hline 10 & 77 & M & T3 N1 M0 & Non-CR & T2 N0 M0 & CR & 2 & 2 & 0 & None & $\begin{array}{l}\text { Death from } \\
\text { other cause }\end{array}$ \\
\hline 11 & 66 & M & T4 N1 M1b & Non-CR & $\begin{array}{l}\text { T1b N0 } \\
\text { M0 }\end{array}$ & CR & 0 & 0 & 0 & LNs & $\begin{array}{l}\text { Death from } \\
\text { ESCC }\end{array}$ \\
\hline 12 & 75 & M & T2 N1 M0 & Non-CR & T1 N0 M0 & $\mathrm{CR}$ & 0 & 0 & 0 & None & Alive \\
\hline 13 & 68 & $\mathrm{~F}$ & T1b N0 M0 & CR & $\begin{array}{l}\text { T1b N0 } \\
\text { M0 }\end{array}$ & CR & 0 & 1 & 0 & None & Alive \\
\hline 14 & 83 & M & T1b N1 M0 & Non-CR & T1 N0 M0 & Non-CR & 0 & 0 & 0 & None & Alive \\
\hline 15 & 69 & $\mathrm{~F}$ & T4 N1 M1b & CR & T2 N0 M0 & CR & 0 & 0 & 2 & None & Alive \\
\hline 16 & 63 & M & T4 N1 M1b & CR & $\begin{array}{l}\text { T1b N0 } \\
\text { M0 }\end{array}$ & Non-CR & 1 & 2 & 0 & Primary site & Alive \\
\hline 17 & 69 & M & T2 N1 M1b & Non-CR & T1 N0 M0 & CR & 1 & 0 & 0 & None & Alive \\
\hline 18 & 77 & M & T4 N1 M0 & CR & $\begin{array}{c}\text { T1b N0 } \\
\text { M0 }\end{array}$ & CR & 0 & 0 & 1 & None & $\begin{array}{l}\text { Death from } \\
\text { other cause }\end{array}$ \\
\hline
\end{tabular}

*Severity was graded according to National Cancer Institute Common Toxicity Criteria (CTCAE), version 4.0. Esophageal stricture: Grade 1; Asymptomatic; clinical or diagnostic observations only; intervention not indicated, Grade 2; Symptomatic; altered GI function. Esophageal obstruction: Grade 1; Asymptomatic; clinical or diagnostic observations only; intervention not indicated, Grade 2; Symptomatic; altered GI function; limiting instrumental ADL. Photosensitivity: Grade 1; Painless erythema and erythema covering $<10 \%$ BSA, Grade 2; Tender erythema covering $10 \%-30 \%$ BSA. CRT: chemoradiotherapy. PDT: photodynamic therapy. CR: complete response. ESCC: esophageal squamous cell carcinoma. LNs: lymph nodes.

tients, N1 M0 in one patient, and N1 M1b (LYM) in one patient. Lymphoid metastases in two patients were evaluated as stable disease at the time of salvage PDT.

\subsection{Response to Salvage PDT}

CR at primary site was achieved by salvage PDT in 11 of 18 patients (61.1\%). The CR rates of the 12 patients with uT1 and the six patients with uT2 were 66.7\% (8/12) and 50.0\% (3/6), respectively. The endoscopic findings of a representative case in which primary CR was achieved by salvage PDT are shown in Figure 3. For a median follow-up of 28.3 months, the PFS rates at 1 and 2 years from the initiation of salvage PDT were 38.9\% (95\% CI, 16.4 - 61.4) and 33.3\% (95\% CI, 11.6 - 55.1), respectively (Figure 4(a)). The OS rates at 1 and 2 years from the initiation of salvage PDT were $77.8 \%$ (95\% CI, 58.6 - 97.0) and 60.6\% (95\% CI, 37.8 - 83.4), respectively (Figure 4(b)). 


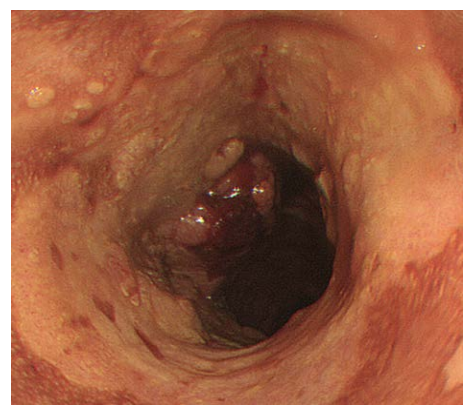

(a)

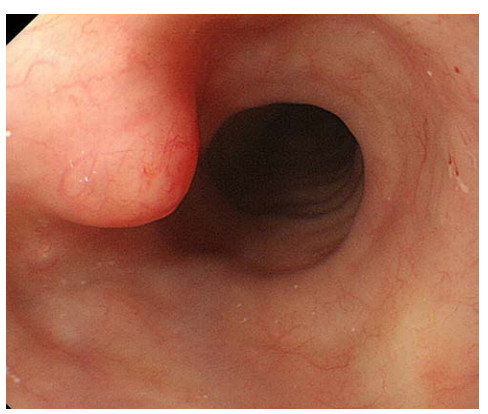

(b)

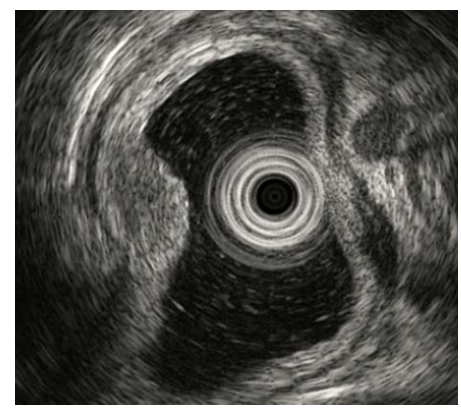

(c)

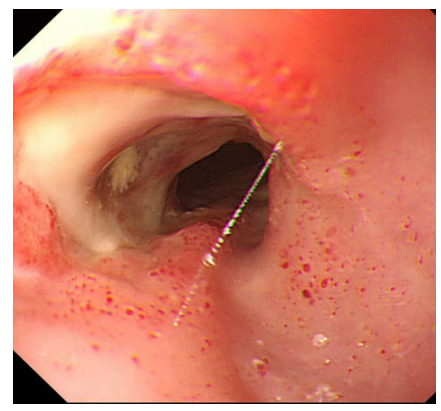

(d)

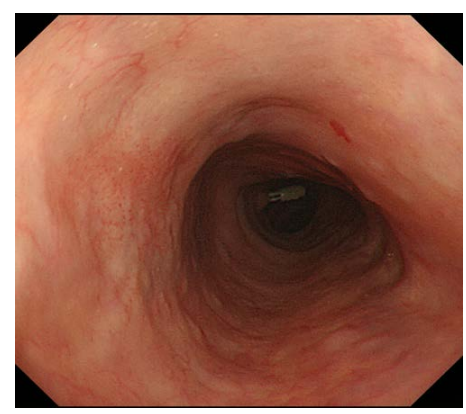

(e)

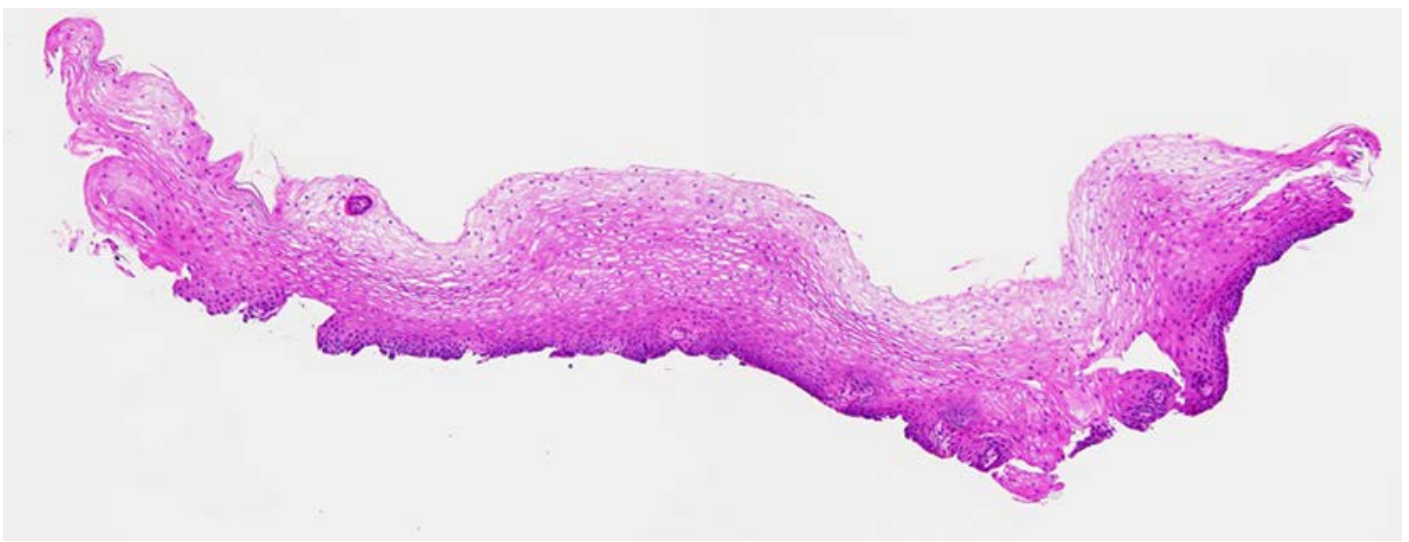

(f)

Figure 3. Representative case who achieved a complete response (CR) after salvage photodynamic therapy (PDT). (a) Advanced esophageal cancer with a circular superficial extensive lesion was detected before chemoradiotherapy (CRT). (b) Although a CR was achieved by CRT for a while, histologically proven local recurrence was detected at the primary site. (c) The depth of the recurrence was evaluated as uT1b with endoscopic ultrasound. (d) At 1 month after salvage PDT, deep ulceration with a mild stricture was observed at the primary site. (e) At 1 year after salvage PDT, the treatment efficacy was evaluated as a CR without any recurrence. (f) Histological finding of one of the biopsy specimens obtained from the primary site at 1 year after salvage PDT. There are no malignant findings in each specimen.

\subsection{Complication of Salvage PDT}

Several complications were observed after salvage PDT. Esophageal stricture requiring endoscopic balloon dilatation was observed in 7/18 (38.9\%) patients even though preventive endoscopic balloon dilatation was performed weekly after the salvage PDT in 9/18 (50.0\%). Esophageal obstruction caused by necrotic tissue was observed in 9/18 (50.0\%) patients. Photosensitivity was observed in 3/18 (16.7\%) patients. The grades were grade 1 (painless erythema) in one patient and grade 2 (painful erythema) in two patients; grade 3 (erythema with desquamation) and grade 4 (life-threatening; disabling) were not observed (Table 1). There were no severe adverse events such as esophageal perforation, mediastinitis, pneumonia, or treatment-related death. 


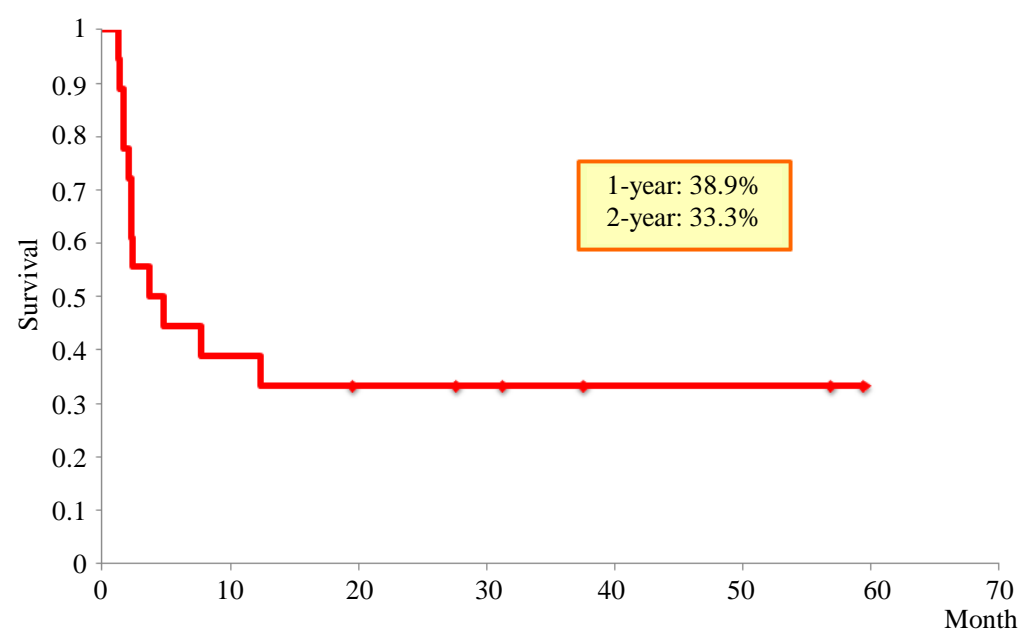

(a)

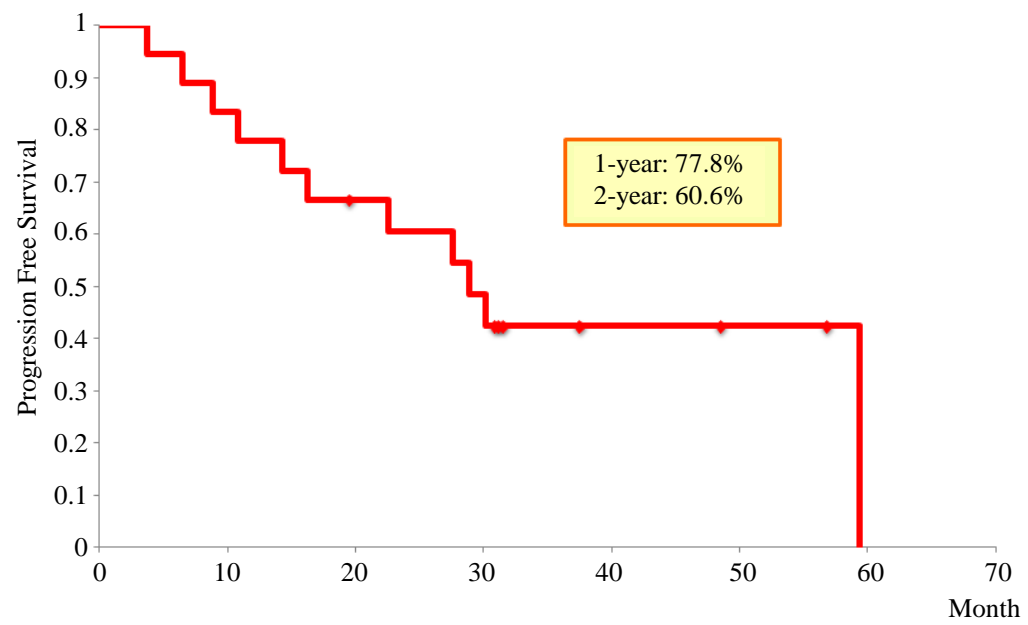

(b)

Figure 4. (a) Progression-free survival of all 18 patients from the initiation of salvage photodynamic therapy. (b) Overall survival of all 18 patients from the initiation of salvage photodynamic therapy.

\subsection{Clinical Course after Salvage PDT}

Five of the 11 patients (45.5\%) who achieved CR by salvage PDT are still alive without any recurrence, two patients (18.2\%) died from ESCC, and one patient (9.1\%) died from another disease. By contrast, seven patients (38.9\%) did not achieve primary CR after salvage PDT. Five of these patients showed regrowth of the local residual tumor; three were treated with PDT again, one was treated with salvage esophagectomy, and one received systemic chemotherapy. Another two of the five patients developed both local residue and lymph node metastases, and received systemic chemotherapy. Two of the seven patients who did not achieve primary CR (28.6\%) are still alive with the disease; five of these patients (71.4\%) died from ESCC. Finally, after a median 28.3month follow-up, five patients are still alive without any recurrence, two patients are alive with the disease, eight patients had died from ESCC, and three patients had died from another disease.

\section{Discussion}

There are some reports of PDT as a first-line treatment for ESCC [6]; however, only a few studies have reported on the use of PDT for salvage treatment for failure of first-line CRT. Yano et al. reported on the efficacy and safety of PDT as a salvage treatment for failure after the CRT for ESCC [7] [8]. In this retrospective analysis, salvage PDT showed a high primary CR rate, which led to high PFS and OS rates 1 and 2 years after the initia- 
tion of salvage PDT. Salvage PDT was indicated for patients who refused a salvage esophagectomy or whose general condition was poor and thus could not receive esophagectomy. The only treatment option other than salvage PDT for these patients was systemic second-line chemotherapy. However, the overall response rate is low and a CR is not expected by second-line chemotherapy after CRT [3] [11] [12]. Considering the poor results of second-line chemotherapy, the high CR rate (61.1\%), PFS rates at 1 and 2 years (38.9\% and 33.3\%) and OS rates at 1 and 2 years (77.8\% and 60.6\%) achieved by salvage PDT seem to be acceptable. In this study, CR rate was higher in patients with uT1 $(66.7 \%)$ than in uT2 patients $(50.0 \%)(P=0.49)$. The OS rate was higher in patients with uT1 than in uT2 patients $(P=0.059)$ (Figure 5). These results suggest that the early detection of a residual or recurrent local lesion might have contributed to the promising result. Therefore, it is important to establish a careful follow-up strategy for patients after CRT. Several mild complications such as esophageal stricture (38.9\%), esophageal obstruction (50.0\%), or photosensitivity (16.7\%) were observed after the salvage PDT, but there was no severe adverse events or treatment-related deaths. For elder patients, especially those with severe coexistent disease, or patients with clinical T4 stage before CRT, salvage surgery is difficult to apply because of the high risks of mortality and morbidity. Although six patients older than 75 years and 10 patients with clinical T4 stage ESCC before CRT were included in this analysis, salvage PDT could undergo without any severe complications. From these aspects, PDT is a safe and effective treatment option provided local failure after CRT for carefully selected patients, such as those with local recurrence or residue without lymph node metastasis, or those whom surgery would be intolerable because of physical complications. At present, EMR/ESD, PDT, and esophagectomy could be a treatment option as salvage treatments for failure after CRT for ESCC. Salvage EMR or ESD was reported as a safe and promising treatment option only for superficial residual or recurrent lesions. Salvage esophagectomy is applied for non-T4 $(\leq \mathrm{T} 3)$ local failure or regional lymph node failure, although it has high risks of mortality and morbidity. The salvage PDT could be indicated for local failure after CRT when the residual or recurrent lesions are suspected at stage T2 or earlier and without lymph node or distant metastasis; that is, salvage PDT takes a mean position between salvage EMR/ESD and esophagectomy. In the present study, 5/18 (27.8\%) patients did not have a histologically proven residual or recurrent tumor before salvage PDT. We cannot deny the possibility that the CR rate might be flattered by these patients. However, even these patients had apparent tumor growth at the primary site and EUS also showed suspicious tumor echo. By contrast, missing the appropriate timing of salvage treatment may decrease the patient's chance of survival. Therefore, we applied salvage PDT as soon as possible for patients with any suggestive findings such as progressive development of ulceration or submucosal elevation evaluated by endoscopic examination and EUS without a histological confirmation of ESCC. The problems associated with porfimer sodium used as a photosensitizer and the excimer dye laser (EDL-1) used as the laser light in this study are as follows: 1 ) the necessity of a long period shielded from light (about 1 month), 2) a high frequency of esophageal obstruction caused by

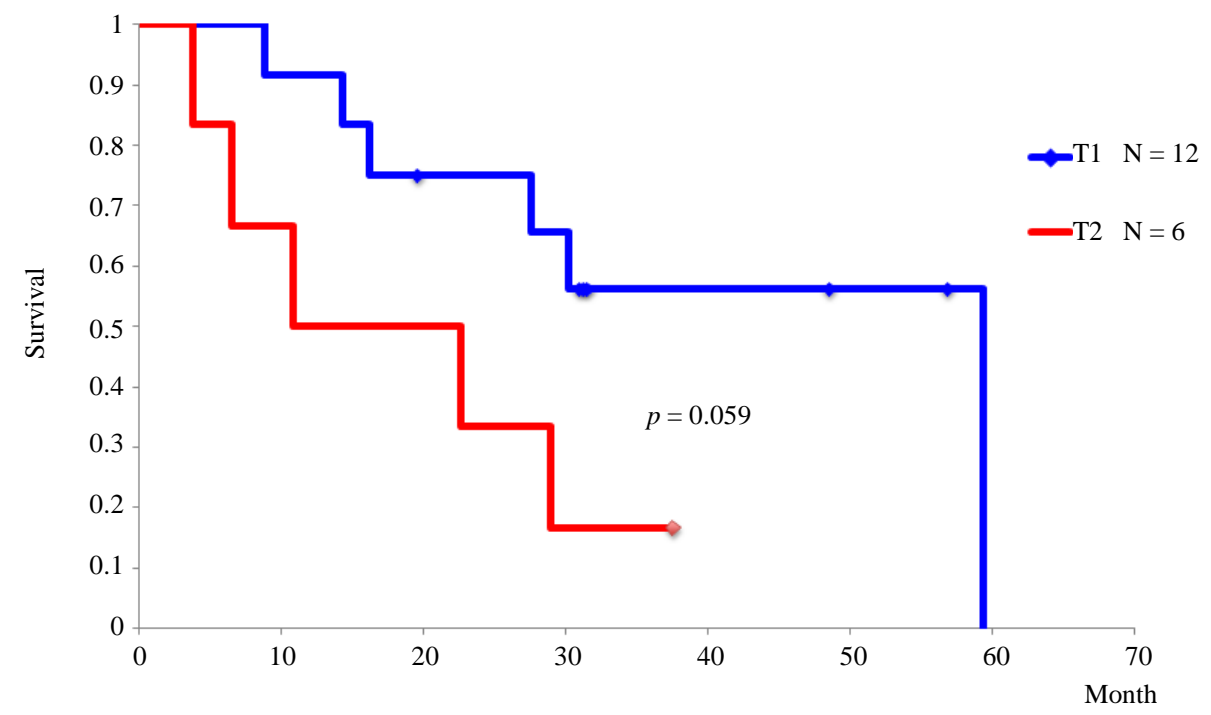

Figure 5. Overall survival of patients with uT1 or uT2 recurrence from the initiation of salvage photodynamic therapy (PDT). 
necrotic tissue, and 3) the cost and the large size of the equipment. The other PDT method using the photosensitizer talaporfin sodium and a diode laser is expected to overcome those problems. A Phase I/II study of PDT using talaporfin sodium and a diode laser as a salvage treatment for local failure after CRT or RT for patients with ESCC (UMIN000003970) is ongoing. Because of the small number of cases (18 cases), relatively short median follow-up period (28.3 months), inclusion of histologically unproven patients, and retrospective design of this study, we cannot draw definitive conclusions about the efficacy and safety of salvage PDT for local failure after CRT for ESCC patients from the results of this work alone. However, the results presented here provide valuable data about the safety and efficacy of salvage PDT because the frequency of patients who have an eligible lesion for salvage PDT is low. We think it is important to accumulate significant evidence about the effects of salvage PDT to generalize for use in daily clinical practice.

\section{Conclusion}

In conclusion, salvage PDT may be a promising treatment option for patients with the local failure after CRT for ESCC when their residual or recurrent lesions are suspected at stage T2 or earlier and without the lymph node or the distant metastasis. It is important to accumulate a greater number of cases to confirm whether salvage PDT is an effective and safe treatment option for local failure after CRT for ESCC.

\section{Conflict of Interest Statement}

No declared.

\section{References}

[1] Herskovic, A., Marts, K., Al-Sarraf, M., Leichman, L., Brindle, J., Vaitkevicius, V., et al. (1992) Combined Chemotherapy and Radiotherapy Compared with Radiotherapy Alone in Patients with Cancer of the Esophagus. New England Journal of Medicine, 326, 1593-1598. http://dx.doi.org/10.1056/NEJM199206113262403

[2] Muro, K., Hamaguchi, T., Ohtsu, A., Boku, N., Chin, K., Hyodo, I., et al. (2004) A Phase II Study of Single-Agent Docetaxel in Patients with Metastatic Esophageal Cancer. Annals of Oncology, 15, 955-959. http://dx.doi.org/10.1093/annonc/mdh231

[3] Swisher, S.G., Wynn, P., Putnam, J.B., Mosheim, M.B., Correa, A.M., Komaki, R.R., et al. (2002) Salvage Esophagectomy for Recurrent Tumors after Definitive Chemotherapy and Radiotherapy. Journal of Thoracic and Cardiovascular Surgery, 123, 175-183. http://dx.doi.org/10.1067/mtc.2002.119070

[4] Onozawa, M., Nihei, K., Ishikura, S., Minashi, K., Yano, T., Muto, M., et al. (2009) Elective Nodal Irradiation (ENI) in Definitive Chemoradiotherapy (CRT) for Squamous Cell Carcinoma of the Thoracic Esophagus. Radiotherapy and Oncology, 92, 266-269. http://dx.doi.org/10.1016/j.radonc.2008.09.025

[5] Yano, T., Muto, M., Hattori, S., Minashi, K., Onozawa, M., Nihei, K., et al. (2008) Long-Term Results of Salvage Endoscopic Mucosal Resection in Patients with Local Failure after Definitive Chemoradiotherapy for Esophageal Squamous Cell Carcinoma. Endoscopy, 40, 717-721. http://dx.doi.org/10.1055/s-2008-1077480

[6] Sibille, A., Lambert, R., Souquet, J.C., Sabben, G. and Descos, F. (1995) Long-Term Survival after Photodynamic Therapy for Esophageal Cancer. Gastroenterology, 108, 337-344. http://dx.doi.org/10.1016/0016-5085(95)90058-6

[7] Yano, T., Muto, M., Minashi, K., Ohtsu, A. and Yoshida, S. (2005) Photodynamic Therapy as Salvage Treatment for Local Failures after Definitive Chemoradiotherapy for Esophageal Cancer. Gastrointestinal Endoscopy, 62, 31-36. http://dx.doi.org/10.1016/S0016-5107(05)00545-6

[8] Yano, T., Muto, M., Minashi, K., Onozawa, M., Nihei, K., Ishikura, S., et al. (2011) Long-Term Results of Salvage Photodynamic Therapy for Patients with Local Failure after Chemoradiotherapy for Esophageal Squamous Cell Carcinoma. Endoscopy, 43, 657-663. http://dx.doi.org/10.1055/s-0030-1256373

[9] UICC (Union for International Cancer Control) (1997) TNM Classification of Malignant Tumors. 5th Edition, WileyLiss, New York.

[10] Tahara, M., Ohtsu, A., Hironaka, S., Boku, N., Ishikura, S., Miyata, Y., et al. (2005) Clinical Impact of Criteria for Complete Response (CR) of Primary Site to Treatment of Esophageal Cancer. Japanese Journal of Clinical Oncology, 35, 316-323. http://dx.doi.org/10.1093/jico/hyi095

[11] Conroy, T., Etienne, P.L., Adenis, A., Wagener, D.J., Pillot, B., François, E., et al. (1996) Phase II Trial of Vinorelbine in Metastatic Squamous Cell Esophageal Carcinoma. European Organization for Research and Treatment of Cancer Gastrointestinal Treat Cancer Cooperative Group. Journal of Clinical Oncology, 14, 164-170. 
[12] Lordick, F., von Schilling, C., Bernhard, H., Hennig, M., Bredenkamp, R. and Peschel, C. (2003) Phase II Trial of Irinotecan plus Docetaxel in Cisplatin-Pretreated Relapsed or Refractory Oesophageal Cancer. British Journal of Cancer, 89, 630-633. http://dx.doi.org/10.1038/sj.bjc.6601168

\section{Abbreviations}

CR: complete response

CRT: chemoradiotherapy

CT: computed tomography

EGD: esophagogastroduodenoscopy

EMR: endoscopic mucosal resection

ESCC: esophageal squamous cell carcinoma

ESD: endoscopic submucosal dissection

EUS: endoscopic ultrasound

LNs/LYM: lymph nodes

non-CR: not achieving a CR

OS: overall survival

PDT: photodynamic therapy

PFS: progression-free survival

$\mathrm{RT}$ : radiation therapy

SCC: squamous cell carcinoma 